\title{
Does Age Influence Loss of Heterozygosity?
}

\author{
Laurie L. Carr and Daniel E. Gottschling* \\ Division of Basic Sciences, Fred Hutchinson Cancer Research Center \\ *Corresponding author: \\ 1100 Fairview Avenue North, P.O. Box 19024, Seattle, WA, 98109, USA \\ E-mail: dgottsch@fhcrc.org Tel: (206) 667-4494 Fax: (206) 667-5939
}

Keywords: Loss of Heterozygosity, DNA repair, Aging, Double-strand break, Tumor suppressor, Non-homologous end joining, Homologous recombination 


\begin{abstract}
The striking correlation between advanced age and an increased incidence of cancer has led investigators to examine the influence of aging on genome maintenance. Because loss of heterozygosity $(\mathrm{LOH})$ can lead to the inactivation of tumor suppressor genes, and thus carcinogenesis, understanding the affect of aging on this type of mutation event is particularly important. Several factors may affect the rate of $\mathrm{LOH}$, including an increase in the amount of DNA damage, specifically double-strand breaks (DSB), and the ability to efficiently repair this damage via pathways that minimize the loss of genetic information. Because of experimental constraints, there is only suggestive evidence for a change in the rate of DNA damage as humans age. However, recent studies in model organisms find that there are increased rates of $\mathrm{LOH}$ with age, and that repair of DNA damage occurs via a different pathway in old cells versus young cells. We speculate that the age-dependent change in DNA repair may explain why there is increased $\mathrm{LOH}$, and that the findings from these model organisms may extend to humans.
\end{abstract}

\title{
1. Introduction
}

Age is the greatest carcinogen - as a person enters middle-age they begin a period in which there is an exponential increase in the incidence of many types of cancer with each additional year (DePinho, 2000). The precise mechanism underlying the dramatic age-related rise of cancer remains unclear, but the importance of mutation in cancer presents a likely explanation (Knudson, 2001). It is hypothesized that a small number of genetic events — causing either activation of oncogenes or inactivation of tumor suppressor genes — precede each stage of tumorigenesis (Armitage, 1954; Armitage, 1957; Knudson, 2001). By middle age, sufficient cellular genomic damage may have accumulated to initiate carcinogenesis.

However, based on the rates of spontaneous mutation observed in human tissue 
culture cells, the steady accumulation of mutations cannot account for the amount of genetic changes that are present in most tumors (Lengauer et al., 1998). This has led to the hypothesis that one of the early steps in cancer progression is a genetic change causing a switch to a higher than normal rate of mutation, creating a 'mutator phenotype' that increases the likelihood of subsequent genetic events (Loeb, 1991)(Loeb and Loeb, 2003)(Nowak et al., 2002). The relationship between advanced age and the incidence of cancer also raises the question: does aging lead to a higher than normal rate of mutation? If so, is there a specific alteration in genome maintenance responsible for this change? Or is it simply the affect of accumulated mutations at a constant rate over time? To address these questions, we will discuss the affect of aging on loss of heterozygosity, a genetic change that has a significant impact on a cell's normal function.

\section{Loss of heterozygosity}

In considering events that may lead to oncogenesis, tumor suppressors have had a major impact on the thinking, leading to a focus on identifying mechanisms that result in loss of gene function (Brown, 1997). This is particularly relevant in the context of heterozygosity, where one functional dominant allele is "covering” the phenotype of a defective recessive allele. Loss of normal allele function could occur by any number of means, including point mutation, recombination (that "convert” the normal copy to the mutant version), or gene deletion (see Figure 1). The importance of this paradigm emerged in the 1980's during studies of tumorigenesis in patients that had a predisposition to retinoblastoma (Cavenee et al., 1983). Patients who were heterozygous at the $\mathrm{Rb}$ locus, with one wild-type allele and one non-functional allele in the $\mathrm{Rb}$ gene, had a high incidence of tumors that had "lost" the wild type allele of Rb in somatic cells. As noted above, there are several mechanisms by which the normal allele of Rb could become non-functional. However the predominant pathways for loss occurred by 
deletion of the wild-type allele due to loss of an entire chromosome or a portion of it (a hemizygous state), or via a recombination event that replaced the wild-type allele with the non-functional, mutant allele from the homologous chromosome (a homozygous state). Consequently, these genetic changes became known as "loss of heterozygosity" (LOH) events. [Due to limits of methods at the time, point mutations, small deletions, short gene conversions, and epigenetic changes could not be detected. These events are not loss of heterozygosity in the true sense of the word. However, they are frequently classified as LOH events.] Clearly, $\mathrm{LOH}$ is not the only mechanism of genomic instability leading to carcinogenesis, however due to its direct link to tumor development and progression, it is important to understand how $\mathrm{LOH}$ is affected by aging.

LOH can arise as the result of chromosome mis-segregation, or a cell's attempt (or inability) to repair DNA damage in the form of a double-strand break (DSB). Cells are equipped with an array of DNA repair pathways to handle such damage (Paques and Haber, 1999). However in some cases the repair results in changes of the genome, which can manifest as $\mathrm{LOH}$. The relationship between aging and $\mathrm{LOH}$ may be considered as two sides of a coin: Does LOH (and genomic instability in general) lead to aging, and/or does aging lead to $\mathrm{LOH}$ ? In this mini-review we will examine whether $\mathrm{LOH}$ changes as a function of age, evaluating evidence (from a representative set of data) for changes in the rate of $\mathrm{LOH}$ and the means by which it occurs.

\section{Is there an increased rate of $\mathrm{LOH}$ with age?}

Due to the striking correlation between advanced age and the incidence of cancer, it has long been thought that the rate of DNA damage and subsequent mutations must increase as an organism ages (Loeb, 1991) Although it is easy to speculate about sources of increased endogenous damage, including oxidative stress, telomere dysfunction, an increase in collapsed replication forks, etc. (DePinho, 2000)(Finkel and Holbrook, 
2000)(Lombard et al., 2005), it is difficult to actually determine whether there is an increased rate (the number of events per unit time, or per cell division) of mutation in old compared to young individuals. To accurately measure rate of $\mathrm{LOH}$, it is necessary to assay for a mutation with each cell division. Due to this experimental constraint, many studies analyzing changes in genome stability with age are only able to provide information on the changes in mutation frequency - which is missing the important component of time.

\section{The Experimental Data}

Early studies performed by P. Stambrook and J. Tischfield, used a novel assay for LOH in human T-lymphocytes from individuals heterozygous for a non-functional adenine phosphoribosyltranferase gene (APRT) (Gupta et al., 1997). These studies demonstrated LOH in somatic cells, predominately due to mitotic recombination. Building on this work, several investigators have measured the frequency of LOH in young cells and contrasted this to the frequency seen in old cells. For instance, mice heterozygous for the Aprt gene (Aprt+/-) were studied for loss-of-function mutations. Cells that undergo $\mathrm{LOH}$ at this locus (Aprt -/-) become resistant to toxic purine analogs such as 8-aza-adenine (8AA). Spontaneous in vivo events were measured by isolating cells from these mice and expanding the cell population in vitro in the presence of 8AA. In one such study, lymphocytes from young heterozygous mice had a loss-of-function, mutant frequency of $8.7 \times 10^{-6}$ (Van Sloun et al., 1998). Further analysis with allele specific PCR demonstrated 70\% of these mutants had lost the wild-type allele. When similar studies were performed on mice older than one year, the mutant frequency had increased by ten-fold, with 90\% of the mutants demonstrating LOH (Wijnhoven et al., 2001). These results are consistent with a more recent study that measured the frequency of mitotic recombination in pancreatic cells of adult mice with an integrated fluorescent 
reporter (Wiktor-Brown et al., 2006). When cells from mice of increased age (67-74 weeks) were examined, the median frequency of cells that had undergone mitotic recombination within the pancreatic tissue had increased as contrasted to young mice (4 weeks). Furthermore, an increase in the frequency of LOH was detected in the progeny of murine neural stem/progenitor cells from mice two months old vs. those two years old (Bailey et al., 2004).

In a study with human subjects, changes in mutant frequency with age were examined in lymphocytes. Mutant cells in this assay were identified by alterations in the HLA-A locus using antibody-complement immunoselection (Morley et al., 1990). Results showed that the total number of mutant lymphocytes increased significantly with age. The geometric mean frequency increased from $0.71 \times 10^{-5}$ in neonates to $6.53 \times 10^{-5}$ in the elderly (Grist et al., 1992). Similarly, stable chromosome aberrations that increased in frequency with increasing age were observed in human peripheral lymphocytes (Vorobtsova et al., 2001). All these studies demonstrate an accumulation of cells that have undergone loss-of-function mutations and/or LOH in mammals of advanced age.

\section{Unresolved Issues}

Although these studies show increases in $\mathrm{LOH}$ with age, and provide intriguing observations for further investigation, they are of limited value because they all presented their data as mutant frequencies. In contrast to a mutation rate, a mutation frequency is limited in terms of the conclusions that can be made, which is especially relevant to our discussion of changes with age (reviewed in Rosche and Foster, 2000). As a cell grows and divides it has a low but nonzero probability to sustain a mutation during its lifetime, and this probability is its mutation rate. After a cell sustains a mutation it can produce a clone of mutants, and the size of this clone will depend upon when in the growth of the cell population the mutation occurred. For instance, if it occurred early in time, the 
mutation will be represented in more cells and have a higher frequency in the total population than if it occurred later in the growth of the population (the population here may be the total cells of an organ). This issue is further complicated when we consider that the mutation may cause a growth (dis)advantage relative to other cells in the population, or that cell division rates and/or survival may change as a function of age. Thus, in order to determine whether there is a difference in $\mathrm{LOH}$ in young and old organisms, it is critical to determine mutation rate. This quantitative information can then provide insight about qualitative changes that occur in an age-dependent manner in particular biological process(es).

In the human lymphocyte studies the authors attempt to address the issue of clonal expansion by identifying clones among the mutants through analysis of the T-cell Receptor (TCR) $\beta$-chain, or by sequence analysis of the mutations among the clonally expanded cells (Morley et al., 1990). Mutant cells that carry identical TCR rearrangements are considered events from a single clone and are not included within the frequency calculations. Nevertheless, at best, this places an upper limit on the frequency value.

Each of the studies above contrasts cells from a young donor to those of an old donor. However, it has been shown that there is a significant variation in the mutation frequency among individuals of similar age (Gupta et al., 1997). Thus it would be ideal to carry out repeated sampling from an individual over time, to provide a better control for age-induced change. Clearly, such an experiment has inherent limitations with human subjects.

\section{Addressing The Issues}

An assay was designed in budding yeast to measure the rate of $\mathrm{LOH}$ with increasing age, and thus addresses several of these unresolved issues (McMurray and 
Gottschling, 2003). The budding yeast, Saccharomyces cerevisiae, is a model organism tractable to studies of age-induced $\mathrm{LOH}$, in part because it undergoes asymmetric division; a yeast mother cell gives rise to developmentally distinct daughter cells for a number of divisions until the mother cell has reached a full replicative lifespan. To determine the replicative lifespan of a budding yeast cell, micromanipulation is used to dissect each daughter away from the mother until she undergoes senescence. Previous studies have used micromanipulation to ask the question: Does the rate of LOH change with increasing age (McMurray and Gottschling, 2003)? Through the course of a mother cell's replicative lifespan each daughter cell was removed and allowed to form individual colonies. In this way, each cell division of the mother is accounted for by analysis of the daughter colonies. These colonies were then scored for LOH by the use of color markers. As the mothers aged, an increase in the rate of LOH occurred in her progeny. The finding of increased $\mathrm{LOH}$ in the progeny of old cells provided the first direct evidence that the rate of genomic instability changes with age.

Other investigators have detected an increase in frequency of mutations in populations of “non-dividing” yeast over time (Madia et al., 2007). Although these observations have been interpreted as evidence for an increased mutation frequency in post-mitotic cells, it also possible there was a selection for cells that entered the nondividing state with mutations. During the course of this "chronological aging” there is a dramatic reduction in cell viability within the population - thus putting enormous selective pressure for cells with mutations; such a scenario has been documented in bacterial cells (Roth et al., 2006).

\section{Does the Ability to Repair DNA Damage Diminish with Age?}

If we accept the premise that there is an age-dependent increase in $\mathrm{LOH}$, then how does this occur? There are two routes by which an increase could arise: more DSB 
DNA damage and/or a change in the repair of DNA damage. Unfortunately, methods to directly measure DNA damage in the form of DSBs are notoriously insensitive and while surrogate markers (modified or localized repair proteins) are available, they are difficult to accurately quantify (reviewed in Takahashi and Ohnishi, 2005). Furthermore, even with accurate quantification of DSBs, it would be unclear whether an increase in DSBs was the result of more intrinsic DNA damage or a failure to accurately repair normal levels of damage. Consequently, there has been a greater focus on asking whether a cell has the capacity to effectively repair DNA damage. For instance, studies of cultured fibroblasts and lymphoblasts, from human donors of different ages, revealed a decreased capacity to repair UV induced DNA damage in old cells (Goukassian et al., 2000; Moriwaki et al., 1996).

\section{The Experimental Data}

Building on the observations of these earlier studies, the ability of old cells to repair DSBs has been analyzed. Normal human fibroblasts were assessed for circularization of a transfected linear plasmid (Seluanov et al., 2004). With this plasmid rejoining assay, it was found that young fibroblasts were more efficient at nonhomologous end joining (NHEJ) than cells which had been passaged multiple times (presenescent cells) or that had ceased dividing (senescent cells). In addition, DNA repair in the senescent cells was more error-prone in that larger deletions of nucleotides were seen at the repair site. Of note, the authors also used the same cell lines transfected with the catalytic component of the human telomerase to create immortalized cells lines. These cells were considered young, despite undergoing over 100 population doublings (at least 30 more than the normal fibroblast senescent cells) because they had not yet stopped proliferating. There was no change in efficiency of NHEJ in the immortalized, nonsenescent cells compared to the young cells of the wild-type cell line. The authors 
conclude that the changes in efficiency and fidelity of DSB repair by NHEJ were a function of cellular senescence and not the replicative age of these cells.

\section{Unresolved Issues}

This work provides direct evidence for a change in the ability to repair DSBs in senescent cells, and provides a good start for determining what changes may occur in DNA repair as cells senesce (Seluanov et al., 2004). However, it is also important to detect other forms of DSB repair, particularly homologous recombination (HR). NHEJ and HR are likely to be in competition for DSB substrates, so a reduction in NHEJ activity may lead to an increase use of homologous recombination pathways, which can increase the amount of $\mathrm{LOH}$ along an affected chromosome (see Figure 1).

While the plasmid rejoining assay has shown robust difference between senescent and young cells, it is based upon transfection of naked DNA template into cells. However DSBs that arise in old cells are not within naked DNA, but in the presence of chromatin. Because modifications to chromatin at the DNA break site have been found to play a role in DSB repair (Morrison et al., 2004), an assay which analyzes repair of an integrated cut site, or spontaneous DSB, may ultimately be needed to assess what changes are most relevant in aging cells (Haber, 2002).

This set of experiments also highlights another important issue in aging-related studies of DNA repair (and aging studies in general) - defining what an 'old cell' is. Are old cells those that have undergone multiple cellular divisions, those that have been collected from a donor of advanced chronological age? In studies of cells collected from donors of advanced age, is there a significant difference between post-mitotic cells, those that can be further expanded in vitro (such as lymphocytes), and germ cells? This question becomes important in studies where discrepancies are noted between the repair 
capacity of these different 'old' cells. This issue is also relevant when drawing conclusions from various model organisms.

\section{Does the Method of DNA Repair Change with Age?}

Although eukaryotic cells predominantly use NHEJ and HR to resolve DSBs, each of these categories, particularly HR, can be further divided into distinct repair pathways. During HR a template provides the missing DNA sequence information for DSB repair. The cell can use the sister chromatid as the template (sister chromatid exchange), or the homologous chromosome (e.g. gene-conversion or crossing-over). The length of the homologous tract can also vary. For example, in break-induced replication (BIR) the tract extends from the break site all the way to the chromosome end. Because each type of repair results in different changes to the broken chromosome, the type of repair used to resolve a DSB can lead to different amounts of LOH (Figure 1). For example, there is no $\mathrm{LOH}$ when a DSB is repaired via sister chromatid exchange, while BIR produces a large segment of $\mathrm{LOH}$ within the repaired chromosome.

Numerous factors influence the choice of repair method, including the stage of the cell cycle. For example, sister chromatid exchange cannot take place in G1 (there is no sister chromatid). In fact, homologous recombination appears to be repressed in G1 (Fishman-Lobell et al., 1992) and NHEJ is preferentially used in this stage of the cell cycle (Aylon and Kupiec, 2005). Cell-type specific regulation of the DNA damage repair

method has also been demonstrated. For example haploid yeast exert mating type control of NHEJ (Frank-Vaillant and Marcand, 2001). In addition alternative pathways for DSB repair can effectively compete with one another at the same break site (Fishman-Lobell et al., 1992; Tutt et al., 2001). As shown in budding yeast and cultured mammalian cell lines, if one repair pathway within a cell is compromised, whether due to the conditions of the DSB or dysfunction in components of the pathway, other repair pathways are able 
to compete for the DNA substrate and change the predominate form of repair used. As this can change the amount of LOH seen in the repaired chromosome, it is important to understand how advanced age affects which repair mechanism is used at a DSB.

\section{The Experimental Data}

The work described previously, in which budding yeast were analyzed for ageinduced $\mathrm{LOH}$, revealed not only an increase of $\mathrm{LOH}$ in the progeny as the mother cell aged, but also a change in the type of repair (McMurray and Gottschling, 2003). Specifically, old mothers predominantly used BIR, a change from crossing over that is normally seen in young cells. Thus both qualitative and quantitative changes are manifested in LOH as yeast cells age.

Studies in Drosophila have also identified a change in the method of DSB repair, in the germ cells of aging males (Preston et al., 2006). In this study a DSB was induced within a reporter construct in premeiotic, male germ cells. The males were mated with different virgin females each week throughout the male's life, and the DNA in the resulting progeny were examined for how the DSB was repaired. As the males aged, there was a switch from predominantly NHEJ to predominantly HR repair. In addition, the length of the homologous recombination tract was longer in the progeny of old than in young male flies. Thus once again, cells of advanced age appeared to have a change in the primary DNA repair pathway; this change resulted in increased amounts of LOH.

\section{Unresolved Issues}

Although these studies provide new evidence for an influence of age on $\mathrm{LOH}$, they also have experimental limitations. While micromanipulation of the daughters of aging yeast allows each division of the aging mother cell to be followed, $\mathrm{LOH}$ can only be analyzed in the progeny with this assay (McMurray and Gottschling, 2003). In this way the genomic instability of the progeny is a surrogate for analysis of the effect of 
aging on the mother's genome. Ultimately, the analysis of chromosomes and DNA damage repair within the aging mother cell is necessary for a better understanding of how this change is manifested.

The analysis of LOH with aging Drosophila also has limitations (Preston et al., 2006). The authors were not able to assess whether changes in $\mathrm{LOH}$ were associated with changes in cell death, decreased viability or reduced fertility of sperm, thus making it difficult to draw conclusions about the overall efficiency of repair. Also, will these findings from germ cells be relevant to what happens in somatic cells? Regardless, this study represents the most direct evidence concerning a change in DNA repair with age.

\section{Conclusions}

Although studies of mammalian cells consistently reveal an increased frequency of mutations with advanced age, the experimental data to date do not clearly demonstrate an age-dependent change in the rate of $\mathrm{LOH}$ in vivo. However evidence from nonmammalian model organisms has provided some of the first evidence that the rate of LOH does indeed increase with age. Several studies demonstrate an overall functional decline in different types of DNA repair with age. Perhaps the most interesting finding in the studies of age-induced $\mathrm{LOH}$, is the evidence for a change in the mechanism of repair used to resolve a DSB in an old cell. To date this has been demonstrated in two model organisms, S. cerevisiae and Drosophila. In both studies the change in repair has led to increased amounts of $\mathrm{LOH}$ at the repair site. Thus the amount of DNA damage and capacity for repair may ultimately be less important than the repair mechanism used when considering the amount of genomic material which has been altered.

Previous work in young cells has demonstrated that different repair pathways can compete for the same DNA lesion (Fishman-Lobell et al., 1992). In effect the DNA end produced at a DSB site is a substrate for which various DNA repair proteins compete. 
There is evidence that a change in the kinetics of one pathway or a functional decline in the proteins of another can alter the outcome for this substrate. Protein oxidation is known to increase with age, and can lead to protein dysfunction (Stadtman, 1992). In addition oxidative modification does not affect the proteome uniformly, in that some proteins are more prevalent targets (Cabiscol et al., 2000). In studies of budding yeast, carbonylated proteins were visualized in single cells and seen to accumulate with increased replicative age (Aguilaniu et al., 2003). Thus protein modifications due to oxidative damage may alter the balance of functional repair proteins present at a DSB and ultimately affect the changes to the broken chromosome after repair.

In summary, our understanding of age-induced changes to LOH remains incomplete, but recent studies have provided exciting new information on this process. It will be important to understand the full dynamic process of DSB repair in real time to understand the affect of aging on the capacity of old cells to resolve DSBs. For instance, are proteins involved in DSB repair prone to age-related damage, and if so, how might this affect their function and expression levels? Ultimately if we are to understand the affect of aging on LOH, we must understand how a DSB arises and is handled in old cells. Fortunately, our knowledge of age-induced LOH is increasing. 


\section{Figure 1. How a Double-Strand Break leads to LOH.}

A spontaneous double-strand break that occurs following DNA replication (S/G2 in the cell cycle), results in different amounts of $\mathrm{LOH}$ depending on how repair occurs. A schematic version of two homologous chromosomes is presented - one red, one blue. Each chromosome is replicated and one of the sister chromatids (\#2) receives a double strand break. The repair process is initiated and the chromatids are segregated (odd numbered chromatids in one cell and evens in the other).

Truncation occurs when the broken chromosome is capped with a telomere at or near the break site. Chromosome Loss will occur if the lack of effective repair leads to non-disjunction of the affected chromosome. (This may also occur without a DSB - e.g. when there are defects in the mitotic apparatus.) Non-Homologous End Joining (NHEJ) may lead to a small local deletion following resection and ligation of the DSB. Recombination mediated mechanisms, such as Gene Conversion result in loss of the affected allele as it is replaced with a copy of the homologous allele. Break-Induced Replication results in LOH by recombination initiated replication from the DSB to the end of the chromosome. Finally, a homologous recombination event may include a Crossover that leads to reciprocal exchange of genetic information and $\mathrm{LOH}$ in both cells after division.

\section{Acknowledgements}

We thank the members of the Gottschling lab for their comments on the manuscript, and the NIH (R01 AG023779) for support. 


\section{References}

DePinho, R.A., 2000. The age of cancer. Nature 408, 248-54.

Knudson, A.G., 2001. Two genetic hits (more or less) to cancer. Nat Rev Cancer 1, 157-62.

Armitage, P., R. Doll, 1954. The age distribution of cancer and a multi-stage theory of carcinogenesis. Br J Cancer 8, 1-12.

Armitage, P.a.R.D., 1957. A two-stage theory of carcinogenesis in relation to the age distribution of human cancer. Br j Cancer 11, 161-9.

Lengauer, C., Kinzler, K.W., Vogelstein, B., 1998. Genetic instabilities in human cancers. Nature 396, 643-9.

Loeb, L.A., 1991. Mutator phenotype may be required for multistage carcinogenesis. Cancer Res 51, 3075-79.

Loeb, L.A., Loeb, K.R.e.a., 2003. Multiple mutations and cancer. PNAS 100, 776-781.

Nowak, M.A., Komarova, N.L., Sengupta, A., Jallepalli, P.V., Shih Ie, M., Vogelstein, B., Lengauer, C., 2002. The role of chromosomal instability in tumor initiation. Proc Natl Acad Sci U S A 99, 16226-31.

Brown, M.A., 1997. Tumor suppressor genes and human cancer. Adv Genet 36, 45-135.

Cavenee, W.K., Dryja, T.P., Phillips, R.A., Benedict, W.F., Godbout, R., Gallie, B.L., Murphree, A.L., Strong, L.C., White, R.L., 1983. Expression of recessive alleles by chromosomal mechanisms in retinoblastoma. Nature 305, 779-84.

Paques, F., Haber, J.E., 1999. Multiple pathways of recombination induced by double-strand breaks in Saccharomyces cerevisiae. Microbiol Mol Biol Rev 63, 349-404.

Finkel, T., Holbrook, N.J., 2000. Oxidants, oxidative stress and the biology of ageing. Nature 408, 239-47.

Lombard, D.B., Chua, K.F., Mostoslavsky, R., Franco, S., Gostissa, M., Alt, F.W., 2005. DNA repair, genome stability, and aging. Cell 120, 497-512.

Gupta, P.K., Sahota, A., Boyadjiev, S.A., Bye, S., Shao, C., O'Neill, J.P., Hunter, T.C., Albertini, R.J., Stambrook, P.J., Tischfield, J.A., 1997. High frequency in vivo loss of heterozygosity is primarily a consequence of mitotic recombination. Cancer Res 57, 1188-93.

Van Sloun, P.P., Wijnhoven, S.W., Kool, H.J., Slater, R., Weeda, G., van Zeeland, A.A., Lohman, P.H., Vrieling, H., 1998. Determination of spontaneous loss of heterozygosity mutations in Aprt heterozygous mice. Nucleic Acids Res 26, 4888-94.

Wijnhoven, S.W., Kool, H.J., van Teijlingen, C.M., van Zeeland, A.A., Vrieling, H., 2001. Loss of heterozygosity in somatic cells of the mouse. An important step in cancer initiation? Mutat Res 473, 23-36.

Wiktor-Brown, D.M., Hendricks, C.A., Olipitz, W., Engelward, B.P., 2006. Agedependent accumulation of recombinant cells in the mouse pancreas revealed by in situ fluorescence imaging. Proc Natl Acad Sci U S A 103, 11862-7. 
Bailey, K.J., Maslov, A.Y., Pruitt, S.C., 2004. Accumulation of mutations and somatic selection in aging neural stem/progenitor cells. Aging Cell 3, 391-7.

Morley, A.A., Grist, S.A., Turner, D.R., Kutlaca, A., Bennett, G., 1990. Molecular nature of in vivo mutations in human cells at the autosomal HLA-A locus. Cancer Res 50, 4584-7.

Grist, S.A., McCarron, M., Kutlaca, A., Turner, D.R., Morley, A.A., 1992. In vivo human somatic mutation: frequency and spectrum with age. Mutat Res 266, 189-96.

Vorobtsova, I., Semenov, A., Timofeyeva, N., Kanayeva, A., Zvereva, I., 2001. An investigation of the age-dependency of chromosome abnormalities in human populations exposed to low-dose ionising radiation. Mech Ageing Dev 122, 1373-82.

Rosche, W.A., Foster, P.L., 2000. Determining mutation rates in bacterial populations. Methods 20, 4-17.

McMurray, M.A., Gottschling, D.E., 2003. An Age-induced Switch to a HyperRecombinational State. Science 301, 1908-1911.

Madia, F., Gattazzo, C., Fabrizio, P., Longo, V.D., 2007. A simple model system for age-dependent DNA damage and cancer. Mech Ageing Dev 128, 45-9.

Roth, J.R., Kugelberg, E., Reams, A.B., Kofoid, E., Andersson, D.I., 2006. Origin of mutations under selection: the adaptive mutation controversy. Annu Rev Microbiol 60, 477-501.

Takahashi, A., Ohnishi, T., 2005. Does gammaH2AX foci formation depend on the presence of DNA double strand breaks? Cancer Lett 229, 171-9.

Goukassian, D., Gad, F., Yaar, M., Eller, M.s., Nehal, U.s., Gilchrest, B.A., 2000. Mechanisms and implications of the age-associated decrease in DNA repair capacity. FASEB 14, 1325-1334.

Moriwaki, S.-I., Ray, S., Tarone, R.E., Kraemer, K.H., Grossman, L., 1996. The effect of donor age on the processing of UV-damaged DNA by cultured human cells: Reduced DNA repair capacity and increased DNA mutability. Mutation Research 364, 117-123.

Seluanov, A., Mittelman, D., Pereira-Smith, O.M., Wilson, J.H., Gorbunova, V., 2004. DNA end joining becomes less efficient and more error-prone during cellular senescence. Proc Natl Acad Sci U S A 101, 7624-9.

Morrison, A.J., Highland, J., J., K.N., Arbel-Eden, A., Greenblatt, J.F., Haber, J.E., Shen, X., 2004. INO80 and g-H2AX Interaction Links ATP-Dependent Chromatin Remodeling to DNA Damage Repair. Cell 119, 767-775.

Haber, J.E., 2002. Uses and abuses of HO endonuclease. Methods Enzymol 350, 141-64.

Fishman-Lobell, J., Rudin, N., Haber, J.E., 1992. Two alternative pathways of double-strand break repair that are kinetically separable and independently modulated. Mol Cell Biol 12, 1292-303.

Aylon, Y., Kupiec, M., 2005. Cell cycle-dependent regulation of double-strand break repair: a role for the CDK. Cell Cycle 4, 259-61. 
Frank-Vaillant, M., Marcand, S., 2001. NHEJ regulation by mating type is exercised through a novel protein, Lif2p, essential to the ligase IV pathway. Genes Dev 15, 3005-12.

Tutt, A., Bertwistle, D., Valentine, J., Gabriel, A., Swift, S., Ross, G., Griffin, C., Thacker, J., Ashworth, A., 2001. Mutation in Brca2 stimulates error-prone homologydirected repair of DNA double-strand breaks occurring between repeated sequences. Embo J 20, 4704-16.

Preston, C.R., Flores, C., Engels, W.R., 2006. Age-dependent usage of doublestrand-break repair pathways. Curr Biol 16, 2009-15.

Stadtman, E.R., 1992. Protein oxidation and aging. Science 257, 1220-4.

Cabiscol, E., Piulats, E., Echave, P., Herrero, E., Ros, J., 2000. Oxidative stress promotes specific protein damage in Saccharomyces cerevisiae. J Biol Chem 275, 273938.

Aguilaniu, H., Gustafsson, L., Rigoulet, M., Nystrom, T., 2003. Asymmetric Inheritance of Oxidatiely Damaged Proteins During Cytokinesis. Science 299, 17511753. 


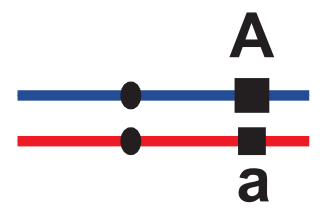

Replication \& DSB
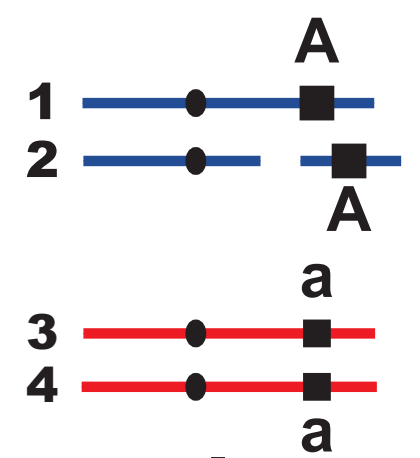

Repair \& Mitosis

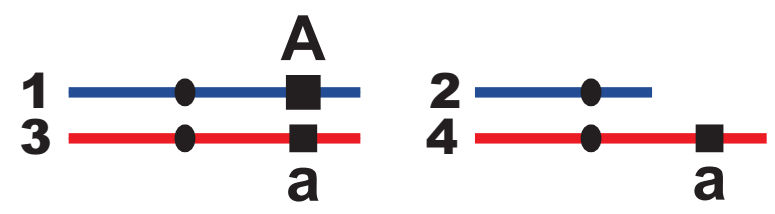

Truncation
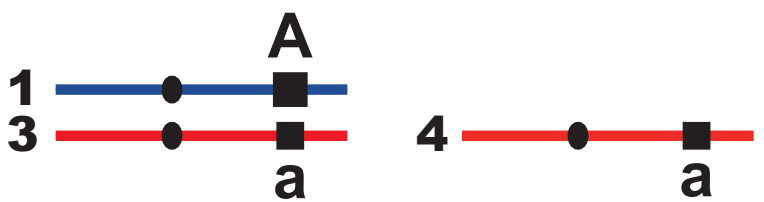

Chromosome Loss
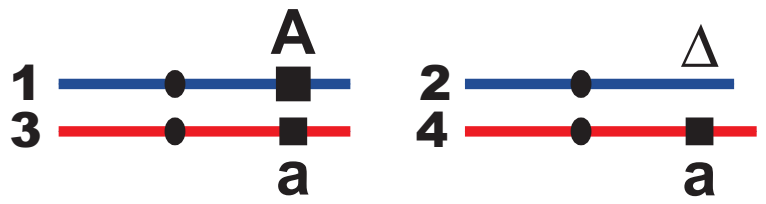

NHEJ with

Local Deletion
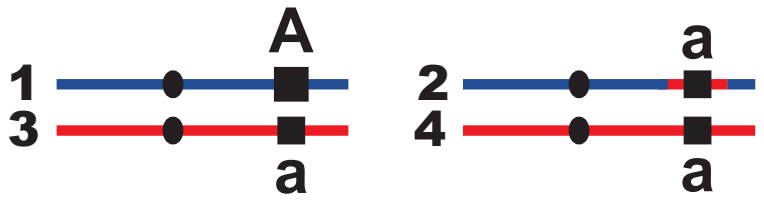

Gene

Conversion
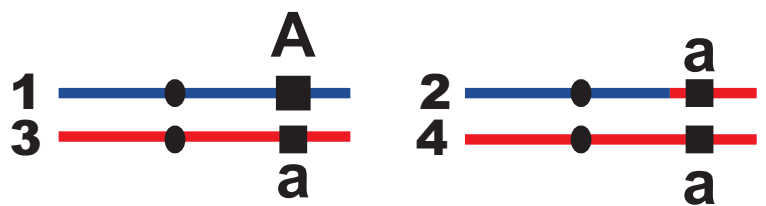

Break-Induced Replication
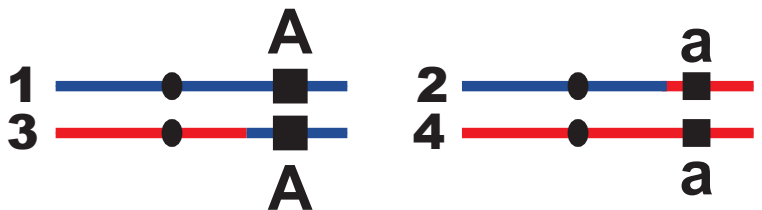

Crossover 